\title{
Inbound Marketing en LinkedIn para la gestión de marca
}

\section{Inbound Marketing in LinkedIn for Brand Management}

\author{
Guadalupe Aguado Guadalupe \\ Profesora Titular del Departamento de Periodismo \\ y Comunicación Audiovisual \\ (Universidad Carlos III de Madrid)
}

Fecha de recepción: 2 de octubre de 2014

Fecha de revisión: 15 de enero de 2015

Para citar este artículo: Aguado Guadalupe, G. (2015): Inbound Marketing en LinkedIn para la gestión de marca, Icono 14, volumen (13), pp. 105-124. doi: $10.7195 /$ ri14.v13i1.741 


\section{Resumen}

El presente trabajo analiza hasta qué punto las prestaciones de Linkedin están orientadas al Inbound Marketing para la gestión de marca, partiendo de que se los usuarios los que llegan a las marcas e interactúan con ellas cuando se produce la necesidad. Para lo cual se requiere de técnicas de comunicación no intrusivas que aporten valor en términos de información, formación, soluciones, experiencia y diversión. Para ello se profundiza en los diferentes usos y herramientas que ha ido desarrollando LinkedIn en su primera y que son esenciales en dicha gestión de marca. Se analiza cómo dicha red ha ido orientando sus prestaciones hacia el marketing de contenidos, el marketing de influencia, el marketing de permiso y el marketing relacional. Se observa cómo se han potenciado estrategias de personalización, buscando la combinación de contenidos relevanes con audiencias relevantes.

\section{Palabras clave}

Gestión de marca - Red social - Marketing de contenidos - Marketing de permiso Marketing de influencia

\section{Abstract}

This study analyzes to what extent LinkedIn's features are geared towards Inbound Marketing for brand management, taking into account that it is the users who the brands reach and who interact with them when the need arises, thus requiring non-obtrusive communication techniques that offer value in terms of information, training, solutions, experience and entertainment. For that purpose, this research conducts an in-depth study of the different uses and tools that LinkedIn has been developing during its first decade and which are essential for said brand management. It analyzes how this network has geared its features toward content marketing, influence marketing, permission marketing and relationship marketing. It can be observed how it has strengthened personalization strategies, seeking the combination of the relevant contents with the relevant audiences.

\section{Key Words}

Brand management - Social network - Content marketing - Permission marketing Influencer marketing 


\section{Introducción}

La gestión de marca como disciplina que engloba todo lo relacionado con el desarrollo de las marcas, de generación de valor a través de estas y la forma en que se consolida su identidad (Homs,2004), ha ido adquiriendo una gran complejidad ante los profundos cambios que han tenido lugar en el comportamiento de los públicos en el nuevo escenario digital, en el que los usuarios (clientes) son los que se encuentran con las marcas e interactúan con ellas de forma consentida y sin interrupciones. Ello ha dado lugar al auge de técnica de marketing no intrusivas que permitan captar seguidores, clientes y prescriptores aportando valor, tal y como señalan Pintado y Sánchez (2014). Al tiempo que se ha ido haciendo necesario dejar de lado la agresividad y el intrusismo, que han sido sustituidos por el permiso, siguiendo las premisas de Godin (1999). Estamos en realidad, tal y como señala Meerman Scott (2007) ante un resultado de la propia evolución del marketing en el ecosistema que las herramientas 2.0 han ido creando en Internet. Lo cual hace que se requieran técnicas no intrusivas que aporten valor en términos de información, formación, comunicación, soluciones, experiencia y diversión.

Ello precisa no sólo de una metodología sino también de una filosofía que influya en la manera en que se gestionan las acciones orientadas a dicha gestión de marca. Teniendo presente que se necesita una comunicación que se base en los principios del Social Media Marketing, explotando la confianza que permiten las relaciones horizontales (Kotler, Kartajaya \& Setiawan, 2011) y la influencia de la inteligencia colectiva, definida por Levy (2004) como la capacidad que tiene un grupo de personas de colaborar para decidir sobre su propio futuro, así como la posibilidad de alcanzar colectivamente sus metas en un contexto de alta competitividad.

Precisamente en dicho contexto ha surgido y ha desarrollado sus diferentes prestaciones la red profesional LinkedIn, que ha ido evolucionando desde su nacimiento en 2002 de red de contactos profesionales hasta convertirse en un espacio de relacionamiento estratégico y gestión de marca (Aguado, 2014). Por ello, el presente trabajo tiene como objeto de estudio las diferentes prestaciones de LinkedIn orientadas a la gestión de marca, que posibilitan el desarrollo de acciones comuni- 
cativas basadas en la metodología del Inbound Marketing, entendido como "todas aquellas técnicas y acciones comunicativas que pretenden llegar al consumidor de una manera no intrusiva en la Red, descartando acciones que molesten al usuario y provocan la interrupción indeseada de su actividad" (Castelló Martínez, 2013:1). Teniendo presente que de entre los pilares del Inbound Marketing la estrategia de contenidos se convierte en la pieza fundamental, tal y como matizan Halligan y Shan (2009). Si bien hay que tener presente, como señala Sanagustín (2013) que el Inbound Marketing es una metodología que combina diversas técnicas de marketing más allá del marketing de contenidos para generar confianza, tales como son el marketing de permiso, que implica la autorización explícita del cliente (Godin, 2001); el marketing relacional, que busca propiciar una relación estable en el tiempo siempre y cuando ambas partes obtengan valor por el mantenimiento de la misma (Barroso Castro \& Martín Armario, 1999) y el marketing de influencia, término que comenzó a usar en los años 60 Daniel Edelman, fundador de la multinacional de Relaciones Públicas Edelman, para denominar el poder que ejercían los famosos sobre los consumidores. Poder que gracias al nuevo entorno digital ha ido abriendo paso a otros sectores.

Igualmente importante a la hora de enfocar las acciones comunicativas es considerar los cinco pilares del Inbound Marketing: atracción de tráfico, conversión, cierre y deleite. Teniendo presente que el Inbound Marketing no sólo se orienta a conseguir clientes finales, sino a mantenerlos satisfechos y cuidar los prescriptores.

El análisis de las diferentes prestaciones y los usos comunicativos que con las mismas se fomentan ayuda a comprender los motivos del éxito de esta red entre profesionales y empresas, tal y como lo demuestran los 300 millones de miembros en 200 países a fecha 2014, los 1,3 millones de propietarios de pequeñas empresas, los 2 millones de ejecutivos a nivel directivo y los 5,5 millones de gerentes de empresas de tecnología. Entre sus usuarios se encuentran los directivos de todas las empresas de la lista Fortune 500. Al tiempo que el 50\% de las empresas de Fortune 100 lo usan. Su acogida se refleja igualmente en estudios como el realizado en enero de 2013 por Wall Street Journal y Vistage International entre 800 Pymes norteamericanas, en el que se recogió que el $41 \%$ de las pequeñas empresas apostaban por LinkedIn como herramienta que posee mayor potencial para su negocio.

ICONO14 | Año 2015 Volumen $13 \mathrm{~N}^{\circ} 1$ | ISSN: 1697-8293 | DOI: ri14.v13i1.741 
La importancia que ha ido adquiriendo LinkedIn en el ámbito profesional y de las organizaciones se ha visto reflejada en informes como How to Use LinkedIn for Business (2010), Learning LinkedIn From the Expert: How to build a Powerful Business Presence on LinkedIn (2012), y (Otras) siete formas de integrar LinkedIn a la reputación online de las compañias (2012).

A esto se une el interés que está adquiriendo el Inbound Marketing, que según refleja el informe State of Inbound Marketing de 2013, realizado por HubSpot, seis de cada diez empresas de marketing de todo el mundo ya han incorporado el Inbound Marketing a sus estrategias.

\section{Objetivos}

\section{El trabajo tiene como objetivos:}

1. Apreciar en qué media la metodología del Inbound Marketing es considerada en el enfoque de las prestaciones de LinkedIn como red profesional orientada a la gestión de marca.

2. Observar en qué grado dichas prestaciones de LinkedIn favorecen usos comunicativos asentados en los principios del marketing de contenido, del marketing de permiso, del marketing relacional y del marketing de influencia.

3. Ver qué prestaciones y usos contribuyen a generar un entorno de confianza, interacción y colaboración, que permita el beneficio de las partes que intervienen en el proceso comunicativo.

\section{Método}

El presente estudio se basa en una metodología cualitativa. Para realizarlo se han seleccionado 14 herramientas que LinkedIn ha desarrollado desde su puesta en funcionamiento en 2002 hasta 2014 y que son esenciales en la gestión de marca: InMaps, botón "sígueme", LinkedIn Skills, LinkedIn Today, LinkedIn Sponsored 
Updates, LinkedIn Showcare, Cube Duel, Mixtent, Linkeduit, Content Marketing Score, Trending Content, LinkedIn Ads, LinkedIn Pulse y Páginas de producto.

Se han considerado igualmente los grupos activos en LinkedIn a fecha de 2014 y se ha observado su funcionamiento, así como la labor de los prescriptores cualificados dentro de los grupos y la importancia de la red de contactos.

Otro aspecto que se ha tenido en cuenta son los rankings que elabora LinkedIn, para ver en qué medida fomentan la competitividad y muestran el posicionamiento.

Se ha observado también el modelo de planificación publicitaria de LinkedIn, tanto en lo que respecta a segmentaciones de mercado como a distribución de anuncios.

Se ha analizado en qué grado dichas prestaciones favorecen los usos comunicativos que se asientan en los principios del marketing de influencia, del marketing de contenidos, del marketing de permiso y del marketing relacional.

Además, se ha considerado en qué medida la comunicación a través de dicha red se basa en los fundamentos y objetivos que inspiran la inteligencia colectiva, en las relaciones horizontales y en las relaciones de intercambio para el fomento de beneficio para las partes.

\section{El valor de los prescriptores para lograr confian- za en un entorno de recomendación cualificada}

La comunicación profesional y de empresas a través de LinkedIn requiere de la socialización de las audiencias, por lo que en la gestión de marca ha de tenerse presente la interacción y la participación de las mismas. De ello se deriva la importancia que desde la puesta en marcha de LinkedIn en 2002 tuvieron los grupos, alcanzando a fecha de 2014 los 1,2 millones de grupos activos. 
Dichos grupos favorecen un entorno comunicativo basado en los principios del marketing de influencia, fomentando relaciones que pueden ayudar a generar visibilidad y cuyo impacto depende de la calidad de los contenidos, confianza y fiabilidad de las fuentes. En este sentido hay que tener presente, como señalan Gratton y Gratton (2012), que la confianza implica convertirse entre otras cosas en solucionador de problemas y guía. Sin perder de vista como matizan Morgan y Hunt (1994) que la confianza implica fe en la fiabilidad e integridad de la otra parte.

Precisamente dicho valor de la confianza hace fundamental en los grupos de LinkedIn a los prescriptores de marca, es decir, aquellos usuarios que dan su opinión sobre individuos, empresas y productos, ayudando a tomar decisiones al resto. Dichos prescriptores contribuyen a formar los llamados grupos de aspiración (Baena Graciá, 2011), o lo que es lo mismo, aquellos grupos y sectores de los que gustaría formar parte a una gran mayoría.

La confianza es un factor que justifica igualmente la importancia que adquiere en los grupos de LinkedIn la red de contactos, no sólo por el número, sino también por la calidad de los mismos, y los beneficios que puedan aportar para las partes. Dicha red de contactos contribuye a crear una marca con la que se acapare la confianza de terceros, ayudando a transmitir credibilidad a través de los valores y percepciones.

En la transmisión de dichos valores y percepciones es necesario considerar que es esencial dentro de la red de contactos el control de los mensajes, ya que "cuanta más información se suministra en el sentido requerido, más probabilidades de éxito se tendrá en hacer coincidir la imagen percibida con la imagen deseada" (Martín García, 2005:66). Ello implica la necesidad de platearse un método que permita la gestión adecuada de las percepciones, recuerdos y expectativas, teniendo muy presente el valor de la interacción. Precisamente este aspecto LinkedIn lo ha tenido en cuenta al dar la posibilidad de escenificar las relaciones a través del servicio InMaps. Ello permite analizar la red de contactos, aportando una foto dinámica de las diferentes partes que la conforman y las relaciones de esos contactos con el resto. $\mathrm{Al}$ mismo tiempo, brinda la oportunidad de conocer la importancia del contacto en lo que a conexiones se refiere, lo que se denomina superconectores de red.

DOI: ri14.v13i1.741 | ISSN: 1697-8293 | Año 2015 Volumen 13 Nº 1 | ICONO14 
En este modelo también se basa LinkedIn a la hora de enfocar la publicidad de las ofertas de trabajo, recomendando éstas a los usuarios en función de sus contactos. En esta modalidad de publicidad, a los usuarios se les presentan ofertas de empresas en las que hay alguno de sus contactos, con el fin de que les puedan dar recomendaciones.

En esta misma línea las páginas de empresa se han convertido en un escaparate que sirve para la recomendación de soluciones, de manera que otros usuarios puedan tomar decisiones en función de lo que sus expertos de confianza hayan valorado. De tal manera que se logra la incorporación de seguidores a la marca, obteniéndose un beneficio mutuo para ambas partes. En el caso de las empresas es una forma de ofrecer su catálogo de productos y servicios de una manera más cercana, con un trato más directo con los profesionales, conociendo de primera mano la valoración de expertos.

Con dicho servicio, LinkedIn refuerza su apuesta de acercamiento a los profesionales, ofreciendo una interesante prestación cuando se quiere conocer una opinión cualificada sobre un servicio determinado, ayudando a la toma de decisiones. Este tipo de prácticas se asienta en un modelo de negocio basado en la colaboración y nos lleva a hablar de una publicidad basada en la recomendación, de manera que los usuarios se convierten en un canal de comunicación en sí mismos. En dicho proceso la inteligencia colectiva es aprovechada para fomentar que los contenidos generados por el público se traduzcan en una optimización del marketing de determinados productos y marcas. Todo ello en un entorno en el que se pasa del poder de los vendedores al poder de los compradores (González; Salutregui \& Sánchez del Toro, 2004), dando lugar a un feedback que permite detectar posibles mejoras, posibilitando a las empresas construir relaciones más estrechas con sus clientes y mantenerlas a largo plazo.

Pero además de fomentar el acercamiento se require dar un paso más, que permita lograr una vinculación a más largo plazo. En este sentido LinkedIn cuenta con el botón "sígueme" para empresas, que muestra el número de seguidores que tiene una determinada compañía. Dicho botón se puede insertar en las páginas web de las marcas permitiendo seguirlas y estar informados de sus actualizaciones. 
Se trata de estrategias que se rigen por los principios del marketing de permiso, consistente en dejar de lado la agresividad y el intrusismo, buscando el permiso del consumidor en el proceso comunicativo (Godin, 1999).

Para conseguir visibilidad y seguimiento no puede perderse de vista que en esta red profesional la reputación se ha convertido en un ingrediente fundamental de la marca, donde lo importante es lo que dicen de ti. Esa reputación depende en gran parte de lo que se comunica y de cómo se establece el diálogo, para lo que es vital convertir a los contactos en presciptores. Téngase en cuenta que en un contexto de sobreoferta, es preciso ganarse la confianza del público, que a su vez viene determinada por la propia fiabilidad que merezca el intermediario, quien ha de actuar como filtro de selección entre los usuarios y la oferta. Esa confianza es especialmente valiosa si se trata de un entorno en el que impera la recomendación cualificada. De tal manera que la credibilidad e incidencia de la información se acrecienta en atención al perfil de los prescriptores y sus respectivas valoraciones. De manera que conocer quién es el creador del contenido confiere credibilidad y autenticidad.

\section{Marketing de contenidos para la captación y fi- delización de usuarios}

Según el estudio B2B Content Marketing Benchmarks, Budgets, and Trends, LinkedIn es la red social considerada más efectiva por los profesionales del marketing para difundir contenidos de Marketing B2B. Este posicionamiento se debe, entre otras cosas, a la evolución del modelo $\mathrm{C} 2 \mathrm{~B}$, por el que los usuarios utilizaban la red para atraer a las empresas y conseguir ofertas de contratación, a modelos B2C y B2B.

La propia evolución de LinkedIn como red profesional en este sentido ha llevado a la necesidad de generar un entorno que permita la captación y fidelización de usuarios, y donde la relevancia del contenido influye en el interés del público. Así pués, dicho contenido ha de ser significativo, teniendo presente que la relación con el usuario gira en torno al contenido, que hace posible la interacción, la co- 
laboración y la fidelización. En dicho contexto, para obtener el resultado deseado es indispensable, como señala Boxwell (1994:15), estar abiertos a aprender de los otros, a identificarlos, estudiarlos y a mejorar en atención a lo aprendido. Por ello, como matiza Codlings (1998:21), es importante mantener una actitud activa, aportando y compartiendo contenido relevante para el público, lo que comporta la toma de decisiones y el paso a la acción, sin perder de vista que lo más importante es mejorar la experiencia del usuario.

A la mejora de dicha experiencia contribuye el perfil profesional y de empresas que impera en LinkedIn, así como las oportunidades derivadas de las relaciones y debates generados en los 1,2 millones de grupos activos en 2014 sobre temas variados, que hacen de esta red un instrumento para la obtención de información cualificada y contactos. Estamos ante una red que permite el rastreo sobre personas, empresas y marcas. Da acceso a información relevante sobre los puestos de trabajo y compañías en que se desempeñan, así como sobre las conexiones laborales entre personas, pues la mayoría de usuarios muestran públicamente sus conexiones. Al mismo tiempo brinda la oportunidad de conocer información sobre la experiencia de una persona, sus áreas de conocimiento y sus proyectos actuales. Todo ello teniendo presente que es una fuente de contacto con expertos que pueden aportar puntos de vista y testimonios, a la vez que posibilita compartir conocimientos y experiencias en diversas áreas de interés. Se puede decir que estamos ante una red que facilita la conexión, la investigación y la obtención de información. Elementos todos ellos indispensable en el marketing de contenidos.

Precisamente para la mejora del rastreo de contenidos se lanzó la herramienta LinkedIn Skills, que permite descubrir tendencias y habilidades en alza en los distintos sectores profesionales. A ello se une la disponibilidad de un cuadro de mando con estadísticas sobre grupos en atención a tres variables: datos demográficos, que posibilitan descubrir si el mercado objetivo se encuentra en dicho grupo de debate; datos de crecimiento sobre el número total de miembros del grupo; y datos de actividad del grupo.

En dicho sentido se ha dado un paso más posibilitando buscar contenidos relevantes en las conversaciones de los grupos para comporbar si son de interés. De

ICONO14 | Año 2015 Volumen 13 Nº 1 | ISSN: 1697-8293 | DOI: ri14.v13i1.741 
esta manera los usuarios tienen la opción de buscar las opiniones de los expertos sobre un tema concreto y una vez se disponga de información suficiente decidir unirse a determinados grupos. Ello evita sumarse a grupos simplemente por el título o la breve descripción. Permitendo al mismo tiempo depurar búsquedas en atención a profesión, categoría e idioma.

Es importante tener en consideración el valor de este tipo de prestaciones en el seguimiento de información referente a empresas. Téngase en cuenta a este respecto que se establece una vinculación directa entre los datos individuales y las empesas en las que se desempeña la actividad profesional. Al tiempo que existen unos dos millones de páginas corporativas en LinkedIn, dando la posibilidad de recibir notificaciones cuando se contrata, despide o asciende a alguien. De manera que se dispone de información sobre vacantes, cambios y ventas de empresas.

La importancia adquirida por el contenido llevó en 2012 al lanzamiento de la aplicación LinkedIn Today, a través de la cual se ofrecían contenidos relacionados con un determinado área de actividad y caracterizados por ser los más compartidos dentro de los grupos de interés en ese sector. Dicha aplicación fue sustituida en 2013 por LinkedIn Pulse, que permite a los usuarios acceder al contenido completo de las noticias y no sólo a un extracto como ocurre en otras plataformas. No sólo se informa de lo que ocurre en un sector profesional, sino que además se produce una selección de los contenidos considerados más valiosos, poniendo la atención en lo que realmente interesa en un determinado ámbito de actividad. Además, Linkedin Pulse ofrece un servicio de contenidos personalizados, adaptados a los intereses profesionales, permitiendo dar a "me gusta" o dejar comentarios. Cuenta a fecha de 2014 con 60 canales diferenciados por temáticas tan diversas como la economía, las finanzas, el deporte, o el entretenimiento, entre otras, de los que catorce superan ampliamente el millón de seguidores y cinco están entre los 3 y 5 millones de seguidores. De esta manera se establece un vínculo estrecho entre los procesos de comunicación y los intereses de los públicos destinatarios.

Los avances de LinkedIn en este sentido se manifiestan en las propias herramientas para medición de efectividad de contenidos, puestas en marcha en 2014. Tal es el caso de Content Marketing Score, que permite saber qué usuarios

DOI: ri14.v13i1.741 | ISSN: 1697-8293 | Año 2015 Volumen 13 Nº 1 | ICONO14 
únicos han interactuado en las páginas de empresa, grupos, actualizaciones de sus empleados, o en los post de influencers. Gracias a esta herramienta la empresa puede saber qué porcentaje de personas ha consultado su contenido, lo ha recomendado, comentado, compartido, o bien ha comenzado a seguir a la empresa. En base a dichos datos se puede elaborar un ranking con resultados de la propia competencia, de forma anónima. Gracias a estos datos las empresas pueden valorar la efectivad de sus estrategias comunicativas en esta plataforma, averiguar qué tipo de acciones son las que registran un mayor alcance y optimizar sus estrategias.

Además, LinkedIn hace sus propias recomendaciones a las empresas para que mejoren posicionamiento. Para ello cuenta con Trending Content, un ranking de los temas más comentados entre el público de una empresa. Se busca identificar tendencias dentro de la propia red social, para lo que clasifican los intereses de los usuarios, de manera que se puedan orientar los contenidos. En dicha clasificación se incluyen los trending topics de LinkedIn y se los asocia con los contenidos clasificados con mayor índice de compromiso e incluso se puede ver qué segmentos de usuarios son los más activos para un tema determinado.

\section{La importancia de la segmentación en la admi- nistración de relaciones para la aportación de valor}

Se puede decir que LinkedIn es un entorno comunicativo en el que se combinan los contenidos relevantes y las audiencias relevantes, permitiendo crear un perfil de comportamiento, que posteriormente entra a formar parte de un público objetivo concreto. Evidentemente ello requiere considerar que se trata de un canal de comunicación, no de ventas, por lo que los usuarios han de ser tratados como interlocutores del mismo nivel, debiendo la marca convertirse en un usuario más de la red para ganarse la confianza del resto. Dicha búsqueda de confianza responde a una filosofía de relación con el usuario, asentada en los principios del marketing relacional (Morgan \& Hunt, 1994), fomentando actividades dirigidas a establecer, desarrollar y mantener con éxito intercambios. 
Para lograr establecer dicha relación es indispensable contar con los medios que permitan diferenciar a los usuarios en atención a perfiles e intereses para identicar sus necesidades y poder aportar valor. Dicha aportación de valor permite establecer relaciones estables, bidireccionales y merecedoras de confianza.

Para lograr el entorno que posibilite dicha relación LinkedIn ha apostado por estrategias de Customer Relations Management (Curry \& Curry, 2000), con especial atención a la segmentación de mercados, en tanto que es importante detectar las diferencias y considerar que cuanto más personal sea el contacto mayor será el impacto. Por ello el objetivo es alcanzar la mayor personalización posible para atraer y fidelizar a los usuarios. No ha de perderse de vista que el Social CRM se basa en establecer y cultivar relaciones de colaboración con clientes para ofrecer valor y una experiencia de lo más personalizada. Tal y como lo define Greenberg (2002 y 2004), es una filosofía y una estrategia de negocio, apoyada en una plataforma tecnológica, que está diseñada para vincular al cliente en una conversación colaborativa que persiga un beneficio mutuo en un entorno transparente y de confianza. De manera que se permite a las organizaciones conocer y conectar con el cliente en términos sociales, con interacciones más relevantes y a través de los canales más adecuados donde el cliente quiere ser contactado.

Ello se refleja en el propio modelo de planificación publicitaria que ofrece esta red social, que se asienta en microcampañas diarias, a ser posible con tres modelos de anuncios diarios por campaña, con diferentes titulares, contenidos e imágenes y un máximo de quince anuncios por campaña que van dirigidas a microsegmentos seleccionados por criterios de geomarketing (Chasco Yrigoye, 2003).

Dicha aplicación de criterios de segmentación geodemográfica permite conocer dónde encontrar espacialmente a los grupos (targets). Esta localización por zonas geográficas se combina con otros datos como tipo de empresa, función profesional, cargo, sector, antigüedad, sexo, ubicación y grupos. Con todo ello se logra una segmentación muy detallada del usuario, identificando a aquellos con intereses en común. Todas las opciones y valores de segmentación son introducidos por usuarios o derivadas algorítmicamente de la información ingresada por dichos usuarios. 
El valor otorgado a la segmentación se aplica igualmente a las estrategias publicitarias de empresas en LinkedIn, pudiendo optar entre la segmentación por cargo, segmentación por profesión y segmentación por sectores de actividad. En dicha línea LinkedIn Ads es una plataforma de publicidad segmentada que permite a pequeños y grandes anunciantes colocar anuncios de texto e imágenes. Para cada anuncio creado la empresa puede incluir hasta 15 variaciones distintas, permitiendo decidir si el anuncio llevará al usuario a la web de la empresa, a un blog 0 página externa a LinkedIn o por el contrario lo lleva a la página o perfil creado en LinkedIn. Se trata de una aplicación que permite segmentar perfiles profesionales al tiempo que ofrece mucha precisión para la publicidad B2B. Se brinda además la posibilidad de seleccionar empresas para hacer publicidad a sus trabajadores, pudiendo dirigirse a puestos concretos y a grupos profesionales o bien a sectores de actividad.

La segmentación se ha llevado también a las áreas de negocio, gracias a las "Páginas de producto" puestas en marcha en 2013. De manera que se pueden hacer actualizaciones según productos o servicios y los seguidores de la empresa pueden segmentar en función de su interés, centrándose en determinadas actualizaciones.

En esta línea se ha creado LinkedIn Sponsored Updates, que brinda la oportunidad de llegar a los usuarios con mensajes menos intrusivos que la publicidad convencional. De esta manera LinkedIn se convierte en una plataforma para la comunicación B2B, donde se fomenta el compromiso de la comunidad y la segmentación del target. A dicha segmentación contribuye LinkedIn Showcare, que permite la segmentación de los canales de comunicación de sus diferentes productos y servicios. De manera que brinda al usuario la opción de poder discriminar qué tipo de información desea recibir.

Dicha segmentación responde a la necesidad de generar un ambiente de confianza, en cuya creación tienen gran importancia los aspectos de carácter comportamental y social, en un entorno en el que el usuario ha de ser canal de comunicación en sí mismo, desempeñando un papel fundamental como prescriptor y altavoz. Por ello, la segmentación de los consumidores ayuda a llegar a una audiencia más útil con menos, buscando un mayor retorno de las inversiones. 
Para el seguimiento de dicho retorno, LinkedIn facilita informes estadísticos en línea en el panel de control de publicidad, de manera que es posible descargarse informes detallados de las impresiones y otras estadísticas de publicidad. No puede perderse de vista que la búsqueda de prestigio y valor de marca llevan a una especial consideración del ROI.

En este sentido LinkedIn evalua la efectividad de los mensajes incluidos tanto en las páginas de empresa como el de los grupos. Las puntuaciones se calculan dividiendo el número de usuarios de LinkedIn que se han relacionado con el contenido de una marca en el último mes por el público objetivo de los usuarios que han participado activamente en ese período mensual.

\section{Herramientas para la evaluación comparativa en un entorno competitivo}

La evolución de los desarrollos de LinkedIn ha ido encaminada a dar respuesta a la búsqueda de una reputación profesional y empresarial en un entorno competitivo y cambiante, así como a aportar el valor añadido de la credibilidad a través de la fiabilidad que merece la opinión de expertos y profesionales.

Para perfilar el marco adecuado que permita establecer redes de influencia, LinkedIn ha apostado por el desarrollo de herramientas, que como en cualquier práctica de networking (Alastruey, 2011) requieren dedicación y constancia para ser efectivas en la mejora de posicionamiento y construcción de un prestigio. Entre dichas herramientas destacan aquellas orientadas a fomentar el benchmarking, entendido como " un proceso continuo y sistemático que permite evaluar comparativamente los productos, servicios y procesos de trabajo de las organizaciones con los mejores del mundo, de forma que la aplicación de esta técnica se transforma en un proceso continuo de aprendizaje y mejora" (Koontz \& Weihrick, 2008:277). Estamos ante un proceso cuyo objetivo es avanzar en los niveles de eficacia y cuya trascendencia no depende de la mera comparación, sino del impacto que pueden tener estas comparaciones sobre los comportamientos encaminados a lograr mejoras y cambios. En todo caso hay que tener en cuenta como indica San Nicolás (2003), que se trata de una herramienta 
estratégica relacionada con la búsqueda acelerada de la competitividad mediante la comparación de lo que hacemos y cómo lo hacemos, con el objetivo de sacar ventaja sobre los mejores. De manera que es esencial en dicho proceso saber seleccionar a aquellos que muestran un desempeño superior, así como reunir y analizar datos que puedan servir de base para las metas del desempeño (Koontz \& Weihrick, 1995).

En dichos principios precisamente se han inspirado algunas de las prestacionas de LinkedIn, como Cube Duel, juego en el que te muestran dos compañeros de trabajo y tienes que elegir con quien trabajas mejor. El resultado es un ranking por empresas donde puedes ver cómo te ven tus compañeros. Además, tienes que jugar para ir liberando empresas en las que has estado anteriormente. En este sentido también se ha lanzado Mixtent, similar a CubeDuel, pero las votaciones se producen por puestos o aptitudes concretas.

Importante igualmente ha sido el desarrollo de herramientas encaminadas a la colaboración entre usuarios y empresas, que se asientan en el caso de LinkedIn en los principios que rigen el Social CRM. En esta línea destacan los desarrollos por parte de terceros en lo que se refiere a comparación de cualidades y competitividad. Es reseñable en este sentido LinkedUit o LinkedOut. Su objetivo es premiar al usuario con mejor perfil en la red social, ya que una vez el usuario se conecta al sitio web se compara y evalúa con alguno de sus contactos, teniendo en cuenta la educación, experiencia, recomendaciones y conexiones, de tal manera que la campaña apela a la naturaleza competitiva del ser humano.

En este ámbito comparativo y de percepciones LinkedIn elabora el ranking The World's Most in Demand Employers, en el que se reflejan las 100 empresas del mundo en las que más empleados querrían trabajar. El ranking de 2013 lo componían empresas de Australia, China, Francia, Reino Unido, Holanda, Alemania, Austria, Suiza, Brasil, India, Canadá y Estados Unidos. En esta misma línea publica el ranking de profesionales influyentes, que en realidad es un escaparate que contribuye a la gestión de marca personal.

Se trata de aplicaciones todas ellas asentadas en los principios de la colaboración e inteligencia colectiva, y donde impera la recomendación y la competitividad.

ICONO14 | Año 2015 Volumen 13 N 1 | ISSN: 1697-8293 | DOI: ri14.v13i1.741 


\section{Resultados y discusión}

El análisis de las diferentes prestaciones seleccionadas para el estudio permite apreciar que las mismas están orientadas al Inbound Marketing para la gestión de marca. Dichas prácticas de Inbound Marketing se han visto favorecidas por el desarrollo de herramientas que posibilitan una comunicación asentada en los principios del marketing de influencia, del marketing de contenidos, del marketing de permiso y del marketing relacional.

A lo largo del trabajo se aprecia que prestaciones tales como el servicio InMaps, las páginas de empresas con recomendación de soluciones, la dinámica de la recomendación de las ofertas de trabajo y el propio funcionamiento de los grupos de debate, se rigen por los principios del marketing de influencia. Todo ello a su vez ha generado un entorno en el que imperan los principios del marketing de permiso, donde se fomenta el seguimiento a largo plazo y la consiguiente fidelización, tal y como se busca con el botón "sígueme".

A ello se ha unido el fomento de estrategias propias del marketing de contenidos, lo cual se ha visto favorecido con prestaciones como LinkedIn Skills, el propio rastreo de contenidos dentro de los grupos y las páginas de empresa. A esto se han sumado prestaciones como LinkedIn Today y LinkedIn Pulse, donde el eje central es el contenido. Se ha llegado incluso a ofertar una herramienta que permite medir la efectividad de dicho contenido, conocida como Content Marketing Score. Al tiempo que se ha desarrollado otra herramienta, Treding Content, que facilita conocer los temas que han despertado mayor interés. Lo cual evidentemente ayuda a la hora de hacer la selección de contenidos a ofertar y el seguimiento de su acogida entre los usuarios.

El Inbound Marketing se ha visto favorecido además por estrategias de segmentación y personalización, siguiendo los principios del marketing relacional, al permitir aportar valor gracias a la diferenciación de los usuarios en atención a perfiles y necesidades. Estas prácticas se han aplicado tanto en campañas publicitarias a través de LinkedIn Ads, como mediante las páginas de productos y de LinkedIn Sponsored Updates, prestación que ofrece la oportunidad de llegar con mensajes

DOI: ri14.v13i1.741 | ISSN: 1697-8293 | Año 2015 Volumen 13 Nº 1 | ICONO14 
menos intrusivos. Es de destacar igualmente en este sentido LinkedIn Showcare, que brinda la oportunidad de discriminar en función de la información que se desea recibir en cada momento por parte de los usuarios.

A todo ello se une la posibilidad de realizar comparaciones entre personas, marcas, empresas y productos para determinar las características que más destacan en cada uno, dando información de valor a la hora de tomar decisiones. Al mismo tiempo, dichas comparaciones permiten a LinkedIn la elaboración de rankings tanto de empresas como de profesionales, con los cuales se fomenta la competitividad, al tiempo que son un elemento más a considerar en la gestión de marca, en cuanto que muestran posicionamiento y número de seguidores.

Se puede decir que estas prestaciones contribuyen a generar un entorno comunicativo en el que es posible establecer una microsegmentación que permite diferenciar intereses de grupo y donde en gran medida la relación e interactividad se generan en torno al contenido. El objetivo es llegar a los usuarios con mensajes no agresivos, que aporten valor y experiencias en un ambiente de confianza. Además, se posibilita el impacto a través de canales por los que el público quiere ser contactado y en el momento temporal en que siente la necesidad de dicho contacto.

Parar ello LinkedIn se sirve de los principios que rigen los fundamentos de la inteligencia colectiva, al tiempo que fomenta la recomendación cualificada y la colaboración, teniendo presente el impacto sobre los comportamientos. Con todo ello se da lugar a un modelo comunicativo en el que impera la calidad de los contactos y el beneficio que aportan para las partes. En dicho entorno el valor de los contenidos se acrecienta en atención al perfil de los prescriptores y sus valoraciones, que contribuyen a generar un ambiente de confianza en el que el usuario se convierte en un canal de comunicación en sí mismo, desempeñando un papel fundamental como prescriptor y altavoz.

\section{Referencias}

Aguado Guadalupe, G. (2014). Gestión de marca en Linkedin: evolución de red de contactos a espacio de relacionamiento estratégico. AdResearch, No 9, Vol. 9, 8-21.

ICONO14 | Año 2015 Volumen 13 Nº 1 | ISSN: 1697-8293 | DOI: ri14.v13i1.741 
Alastruey, R, (2011). El networking. Barcelona:UOC.

Baena Graciá, V. (2011). Fundamentos de marketing: entorno, consumidor, estrategia e investigación comercial. Barcelona: UOC.

Barroso Castro, C. \& Martín Armario, E. (1999). Marketing relacional. Madrid: ESIC.

Boxwell, R. (1994). Benchmarking: para competir con ventaja. Madrid: McGrawHill.

Castelló Martínez, A. (2013). La estrategia de medios sociales, el Inbound Marketing y la estrategia de contenidos: marketing de atracción 2.0. I

Congreso Internacional de Comunicación y Sociedad Digital. Logroño. Chasco Yrigoye, C. (2003). El Geomarketing y la distribución comercial. En Investigación y Marketing, $\mathrm{n}^{0}$ 79, 6-13.

Content Marketing Institute (2014). B2B Content Marketing Benchmarks, Budgets, and Trends. North America.

Curry, J. \& Curry, A. (2000). CRM: Customer Relationship Management. Barcelona: Gestión 2000.

Godin, S. (1999). Permision Marketing: Turning Strangers into Friends, and Friends into Customers. New York: Simon\&Schuster.

Codling, B. (1998). Benchmarking. Madrid: AENOR.

González, J., Salutregui, J. \& Sánchez del Toro, J. (2004). Más allá del CRM y el

marketing relacional: personalización. Madrid: Prentice Hall.

Gratton, S. \& Gratton, D.A. (2012). De 0 a 100.000 Social Media: Para

profesionales y pequeñas empresas. Madrid: Anaya Multimedia.

Greenberg, P. (2002). CRM at the Speed of Light: Capturing and Keeping Customers in Internet Real Time, $2^{\mathrm{a}}$ ed. Berkeley: McGraw-Hill/Osborne.

Greenberg, P. (2004). CRM at the Speed of Light: Essential Customer Strategies for the 21 st Century. New York: McGraw-Hill/Osborne.

Hallian, B. \& Shan, Dh. (2009). Inbound Marketing: Get Found Using Google, Social Media, and Blogs. New Jersey: Wiley \& Sons.

Homs, R. (2004). La era de las marcas depredadoras. México: McGraw Hill Interamericana.

Hubspot (2010). How to Use LinkedIn for Business. Recuperado de http://www. hubspot.com/Portals/53/docs/linkedin.for.business.pdf Hubspot (2012). Learning LinkedIn From the Expert: How to build a Powerful 
Business Presence on LinkedIn. Recuperado de http://cdn2.hubspot.net/ hub/53/blog/docs/ebooks/learning_linkedin_from_the_experts.pdf Hubspot (2013). State of Inbound Marketing. Recuperado de http://cdn2. hubspot.net/hub/53/file-30889984-pdf/2013_StateofInboundMarketing_ FullReport.pdf

Inboundcycle (2013). Linkedin para empresas. Recursos y consejos para una estrategia online eficaz.

Koontz, H. \& Weihrick, H. (2008). Essentials of Management: An International Perspective, $6^{\text {a }}$ ed. New York: Mc Graw-Hill.

Kotler, Ph., Kartajaya, H.\& Setiawan, I. (2011). Marketing 3.0. Madrid: Lid Editorial Empresarial.

Lévy, P. (2004). Inteligencia colectiva: por una antropología del ciberespacio. Recuperado de http://inteligenciacolectiva.bvsalud.org/ LinkedIn (2013). The World's Most in Demand Employers. Recuperado de https:// www.linkedin.com/indemand?_mSplash=1

Llorente \&Cuenca (2012). (Otras) siete formas de integrar LinkedIn a la reputación online de las compañias. Informe especial. Madrid.

Martín García, M (2005). Arquitectura de marca. Modelo general de construcción de marca y gestión de sus activos. Madrid:ESIC.

Meerman Scott, D. (2007). The New Rules of Marketing and PR. New Jersey: John Wiley \&Son, Inc.

Morgan, R.M. \& Hunt, S.D. (1994). The Commintment-Trust Theory of Relationship Marketing. Journal of Marketing, 58/3, 20-38.

Pintado Blanco, T. \& Sánchez Herrera, J. (2014). Nuevas tendencias de comunicación estratégica, $3^{\text {a }}$ ed. Madrid: ESIC.

Sanagustín, E. (2013). Marketing de contenidos. Madrid: Anaya Multimedia.

Santesmases Mestre, M. (2012). Marketing: Conceptos y estrategias. Madrid: Pirámide.

San Nicolás, R. (2003).Lo que el benchmarking puede hacer por su empresa. Ejecutivos de Finanzas. Instituto Mexicano de Ejecutivos de Finanzas, $\mathrm{N}^{\circ} 6$, año XXXII, 44-47. 